\title{
Laser modification of liquid nutrient medium and antibiotic solutions with the assessment by the bacteria growth dynamics
}

\author{
Alexander N. Malov ${ }^{1}$, Anna V. Neupokoeva ${ }^{2 *}$, and Lubov A. Kokorina ${ }^{2}$ \\ ${ }^{1}$ Irkutsk National Research Technical University, 83 Lermontova Str, Irkutsk 664074, Russia \\ 2 Irkutsk State Medical University, 1 Krasnogo Vosstaniya Str., Irkutsk 664009, Russia \\ * e-mail: annett 2005@inbox.ru
}

\begin{abstract}
The processes of laser photoinduced modification of nutrient media and antibiotic solutions are considered. It is shown experimentally that the growth of colonies number is slower on the irradiated medium. The impact of laser radiation on the antibiotic activity is also studied experimentally. It is found that the laser-induced photomodification can affect the antimicrobial activity of the medicinal preparations. (C) 2017 Journal of Biomedical Photonics \& Engineering.
\end{abstract}

Keywords: Bacteria, antibiotic, Laser activation, Culture medium.

Paper \#3149 received 9 Jan 2017; revised manuscript received 3 Mar 2017; accepted for publication 6 Mar 2017; published online 1 Apr 2017. doi: 10.18287/JBPE17.03.020303. [Special Issue. Workshop "Biophotonics" of the XIII all-Russian Youth Samara conference-contest on optics and laser physics].

\section{References}

1. Laser Therapy and Prophylactics, A.V. Kartelishev et al. (Eds.), Practical Medicine, Moscow (2012) [in Russian].

2. T. I. Karu, L. V. Pyatibrat, S. F. Kolyakov, and N. I. Afanasyeva, "Absorption measurements of cell monolayers relevant to mechanism of laser phototherapy: reduction or oxidation of cytochrome c oxidase under laser radiation at $632.8 \mathrm{~nm}$," Photomedicine and laser surgery 26(6), 593-599 (2008).

3. N. A. Tolstoy, and A. A. Spartakov, Electro-Optics and Magneto-Optics of Disperse Systems, Saint-Petersburg University Publishers, Saint-Petersburg (1996) [in Russian].

4. A. V. Finkelshtein, and O. B. Ptitsyn, Protein Physics, Book house "University", Moscow (2002) [in Russian].

5. V. D. Lakhno, Clusters in Physics, Chemistry, Biology, Regular and chaotic dynamics, Izhevsk (2001) [in Russian].

6. A. N. Malov, and A. A. Vaichas, Laser Control of Biomineral Formation. Physical Principles and Prospects of Developing New Medical Nanotechnology, Palmarium Academic Publishing, Saarbrucken, Deutschland (2016). ISBN 978-3-659-60476-8 [in Russian].

7. A. N. Malov, A. Yu. Seteikin, A. V. Neupokoeva, E. S. Musatova, I. E. Golub, L. V. Sorokina, V. S. Fetschenko, and A. A. Vaichas, "The laser radiation action on the biological objects," Optik 124(23), 6034-6041 (2013).

8. R. A. Paringer, A. V. Kupriyanov, and N. Y. Ilyasova, "Dendritic crystallogram images classification," Journal of Biomedical Photonics \& Engineering, 1(2), 135-138 (2015).

9. A. N. Malov, A. V. Neupokoeva, and A. Yu. Sambyalova, "Diagnostics of liquid properties by the example of bioorganic solution," Journal of Biomedical Photonics \& Engineering 2(1), 010304 (2016).

10. A. N. Malov, A. V. Neupokoeva, E. M. Kudryashova, "Craquelure structure of protein film modification under the laser radiation of different spectral range action," Journal of Biomedical Photonics \& Engineering 2(1), 010307 (2016).

11. Determination of antimicrobial activity of antibiotics using the method of diffusion into agar. State Quality Standard of Medical Agent. General Pharmacopeia Article, (GPA replace to GP XII, part 1, GPA 42-006807), Ministry of Health of Russian Federation (2010) [in Russian]. 


\section{Introduction}

The experimental studies devoted to the applications of laser radiation for medical purposes are intensely carried out from the beginning of 1970-s. To date a huge amount of data is accumulated that allows the conclusion that the laser irradiation of blood or other tissues causes the changes, which can be assessed quantitatively by the biochemical indicators of blood, by the angiospasm, and by the time of different wound healing [1]. However, in spite of the large number of techniques of using the laser radiation in medicine, the most part of researchers consider the human organism as a black box, in which the action having a certain intensity and duration produces a certain standard response. The studies devoted to the mechanisms of laser impact are few, which is explained both by the complexity of the studied system, and by the multilayer structure of the response.

Nevertheless, there are three key ideas that should be taken into account in the description of the laser biostimulation mechanism.

First, it is known that the therapeutic effect of laser radiation weakly depends on the wavelength [2]. The first studies were devoted to the application of radiation from helium-neon laser (red light), then, with the appearance of available semiconductor sources the infrared radiation began to be widely used, and at present there are papers on the application of blue and green light. Therefore, it is necessary to decide, whether there is some unique acceptor in the organism that possesses such wide absorption range, or there are many substances, able to absorb and transform the energy of laser radiation, and an individual acceptor is relevant in each particular case.

Second, it is experimentally shown $[1,2]$ that the local impact of laser radiation leads to the response of the entire organism rather than is restricted to a local response, which is traced in the biochemical indicators of functioning of all systems. Therefore, there should exist something responsible for the generalisation of the impact.

Third, the studies devoted to the impact of laser radiation on the living objects of different organisation level (bacteria, plants, animals, humans) show, that a recordable response is observed in all cases. Therefore, the mechanism of laser interaction with the biological objects should possess considerable universality

With the above specific features taken into account, we can suppose that the laser radiation is able to interact with organic macromolecules, modifying their spatial structure (conformation). In the human organism, the laser radiation action to the protein molecules that can changes their second- third- and fourth-order structure, and not affecting the first-order structure, i.e., the sequence of amino acids.

It is known that the reactivity of a macromolecule is directly related to its spatial structure, which is determined by the presence of an active centre, the interaction of the macromolecule with its aqueous environment, and the interaction with other macromolecules [3]. At present it is proved that the substances diluted by the liquids form associates of molecules at the expense of the electrostatic interaction forces $[4,5]$. The molecules included in an associated lose its mobility and the active centres can overlap with the adjacent molecules. The larger size of the associate, the larger ratios of its mass to surface and, therefore, the molecule have to weaker processes of assimilation and reactivity.

In the frameworks of this concept, the laser radiation is absorbed by the associate rather than by a single molecule, which causes the reformation and decay of the associate into smaller fragments. Since in any multicomponent solution the associates of different configurations are formed, they can absorb the energy in a very broad range, which explains the similarity of effects arising under the laser impact with different wavelength.

On the other hand, if the interaction occurs at the "radiation-associate" level, then its results must the observable not only in living systems, but also in model media, such as solutions of proteins, blood plasma, biological liquids, organic solutions for growing bacterial cultures, etc. In Refs. [6-10] it is shown that in relation to the protein and bile solutions this statement finds its experimental confirmation.

The purpose of the present work was to determine the impact of laser radiation on the recordable changes in organic solutions by the example of nutrient media used for bacteria cultivation and the antibiotic solution.

\section{Experiment}

\subsection{Materials and methods}

Two series of experiments were carried out. In the first series, we assessed the influence of the laser irradiation on the nutrient medium by the dynamics of the bacterial growth. In the second series, we studied the impact of laser radiation on the antibiotic activity.

To study the dynamics of the Bacillus subtillis microorganism growth the continuous cultivation was used. The microbial suspension was prepared from the single-day culture grown on the meat peptone agar (MPA) at the temperature of $37 \mathrm{C}$.

Then $1 \mathrm{ml}$ of microbial suspension was added to 100 $\mathrm{ml}$ of nutrient medium cooled to $45 \mathrm{C}$, the components were mixed and poured into three Petri dishes. After the agar hardening all dishes were put into a thermostat at the temperature $37 \mathrm{C}$. The assessment of microbial growth was performed every 12 hours by counting the number of colonies, starting from the zero point. The colonies of microorganisms grown in the nutrient medium without laser irradiation were used as control samples.

The semiconductor laser diode served as a radiation source. The laser impact on the nutrient medium in the regime of "light boiler" was implemented before the addition of the suspension of microorganisms. The exposure time was 120 and 300 seconds, the power of 
radiation was about $100 \mathrm{~mW}$, the wavelength was 532 $\mathrm{nm}$, and the total irradiation energy was nearly $5 \mathrm{~J} / \mathrm{g}$.

In the second series the antibiotic Vancomycin irradiated by the laser and not exposed to laser radiation were added to the nutrient medium using the method of diffusion into agar. Using a sterile cylinder with the diameter $0.5 \mathrm{~cm}$, three holes were made in the dishes with the hardened medium at equal distance from each other and from the edges of the dish. Then the dishes were grouped in pairs, and the holes were filled with physiological solution (one hole in the first dish and one hole in the second dish), the non-exposed antibiotic (two holes in the first dish), and the antibiotic exposed during 120 seconds and 300 seconds (two last holes in the second dich). In 24 hours, we measured the diameters of the zones of Bacillus subtillis growth inhibition with the accuracy to $1 \mathrm{~mm}$ using the millimeter paper, which is a standard procedure for the assessment of antibiotic activity [11].

\subsection{Results and discussion}

In the first series of experiments that allow the assessment of the impact of the laser irradiation of the nutrient medium on the bacterial growth dynamics, the first growth was observed in 12 hours after the experiment zero point. The number of colonies in the control samples and in the laser exposed ones was practically the same. In 24 hours from the zero moment the number of bacteria grown in the control dishes was greater and amounted to $31 \mathrm{CFU}$, while after the $120 \mathrm{~s}$ of laser irradiation it was smaller by $29 \%$ and after the $300 \mathrm{~s}$ exposure it was by $16 \%$ smaller. In 48 hours after the zero point the number of colonies in the control samples amounted to $48 \mathrm{CFU}$, in the samples irradiated during 120 seconds it was smaller by $6 \%$ (45 CFU) and for those irradiated during 300 seconds it was smaller by $17 \%$ (40 CFU). Figure 1 presents the curve of Bacillus subtillis growth in the control sample and in the medium exposed to the laser irradiation. The spread of colony numbers does not exceed $12 \%$ of the presented mean values.

In the second series, we studied the impact of laser radiation on the antibiotic activity by measuring the zones of suppressed growth. The measurements were performed in 24 hours after placing the nutrient medium with the bacterial culture into the thermostat. Around the holes filled with physiological solution for control, we observed intense growth of bacteria. For the unexposed antibiotic, the inhibition zone was $38 \mathrm{~mm}$. For the antibiotic exposed to the laser radiation during 120 seconds, the inhibition zone was $42 \mathrm{~mm}$, which is by $10.5 \%$ greater than without the laser exposure. For the antibiotic exposed during 300 seconds, the growth inhibition zone was $13 \mathrm{~mm}$, i.e., greater by $13 \%$ as compared with the intact antibiotic. The spread of the determined zone size did not exceed $8 \%$ of the mean values.

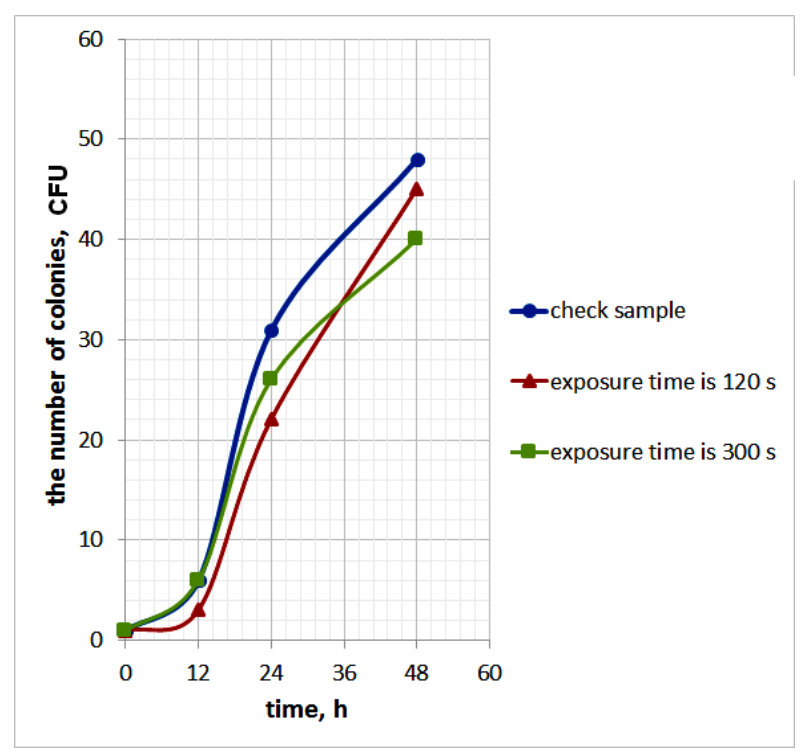

Fig. 1 Curves of Bacillus subtillis growth in the control samples and after the laser irradiation during120 and 300 seconds.

The mechanism of the observed effects can be thought as follows. As mentioned above, the organic molecules in solutions form associates mainly stabilised by hydrogen bonds. Besides that, on the surface of an associate the coating of water molecules is formed, which additionally stabilises the system. In the absence of external perturbations, the associate is rather stable. However, since the energy of its bonds is not high, even a small external perturbation can bring it out of the equilibrium position. In our experiment, the laser radiation deliver the energy that makes the parts of the associate move, and this energy is sufficient to bring the structure out of equilibrium, break the present hydrogen bonds and lead to its decay.

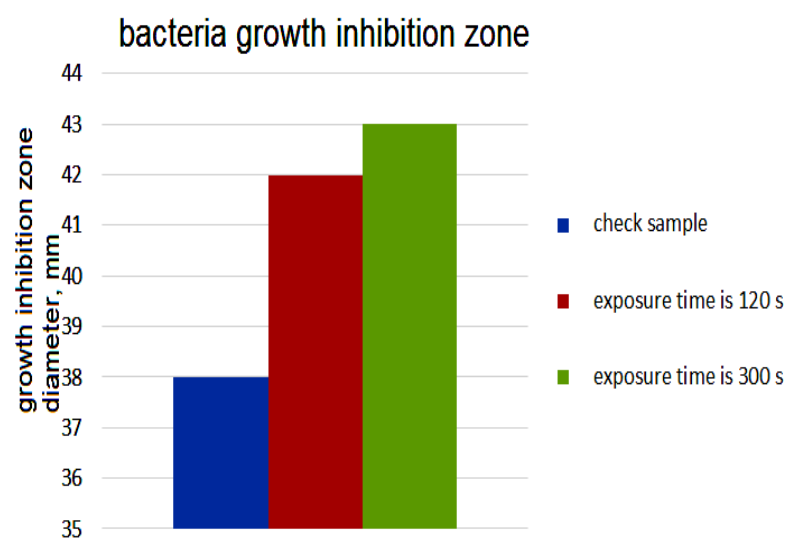

Fig. 2 Zones of Bacillus subtillis growth inhibition for the intact antibiotic and the antibiotic irradiated during 120 and 300 seconds.

In the experiment with antibiotic, the activation effect observed in the experiment is explained as follows. The Vancomycin antibiotic acts because its molecule is bound to the bacterial membrane (or to the 
proteins on the membrane surface). Ideally, each molecule must be bound to one bacterium. However, since the antibiotic is stored in the form of a powder and solve in water or physiological solution immediately before the injection, the solution contains associates rather than single molecules. As a result, the efficiency of binding the antibiotic to bacteria is reduced, since a few molecules are bound to one bacterium instead of many bacteria. One can suppose that under the impact of laser radiation the associates are broken into smaller fragments, so that the reactivity of the antibiotic is increased, and, therefore, its efficiency is increased, too.

The data in Fig 2 can serve as a base for the standard determination of antimicrobial activity of antibiotics using the method of diffusion into agar after the laser impact [11].

\section{Conclusion}

Thus, it is experimentally found that the laser irradiation essentially affects the structure of a liquid, disintegrating the molecular associates. This changes the reactivity of molecules in the solution. In particular, the laser irradiation can increase the antimicrobial activity of antibiotics, as well as affect the parameters of bacterial growth in the irradiated nutrient medium. 